\title{
Сравнительная характеристика
}

клинико-иммунологического статуса пациентов
с аутоиммунным тиреоидитом и его сочетанием
с сахарным диабетом 1 типа

Я.В. Гирш, Л.А. Болотская, А.А. Тарлюн

БУ ВО ХМАО-Югры «Сургутский государственный университет»; Россия, г. Сургут

\section{PЕЗЮME}

\begin{abstract}
Цель исследования: на основании комплексного определения клинических, гормональных и иммунологических маркеров дать сравнительную оценку статуса пациентов с аутоиммунным тиреоидитом (АИТ) и АИТ в сочетании с сахарным диабетом 1 типа (СД1). Дизайн: открытое сравнительное исследование с параллельными группами.

Материалы и методы. В исследование включены 175 пациентов с верифицированным диагнозом АИт: группа 1 - пациенты только с АИТ, группа 2 - пациенты с АИТ и СД1. Проведены комплексный анализ данных анамнеза, клинических проявлений, определение аутоантител (АутоАТ) к тиреоглобулину (ТГ), тиреопероксидазе (ТП0), рецептору тиреотропного гормона (ТТГ), гормонам щитовидной железы, инсулину, а также уровней иммуноглобулинов сыворотки крови.

Результаты. При комплексном обследовании пациентов с АИТ с манифестным гипотиреозом найдены прямые корреляционные связи между содержанием АутоАТ (к ТП0, инсулину, ТГ) и уровнем ТТГ, более выраженные в группе пациентов с АИТ и СД1. Прослеживалась взаимосвязь увеличения содержания ТTГ и роста концентраций иммуноглобулинов сыворотки (IgG, IgM, IgA) в исследуемых группах с закономерным усилением связи в группе больных АИТ и СД1. Оценка клеточного звена иммунитета в группах позволила выявить обратные корреляционные связи уровней ТTГ и CD3+/CD4+ (T-хелперов), CD3+/CD8+ (Т-цитотоксических), более сильные у пациентов с АИТ и СД1. В этой группе значимое повышение количества лимфоцитов CD3+/CD16/56+ имело дополнительно прямую корреляционную связь с уровнем ТТГ.

Заключение. Формирование гипотиреоидного состояния неизбежно приводит к увеличению продукции АутоАТ к ряду белковых структур. Снижение количества CD3+/CD4+ и CD3+/CD8+ связано c их перераспределением в очаги аутоиммунного воспаления, а значительное возрастание относительного и абсолютного количества Т-клеточной субпопуляции (CD3+/CD $16 / 56+)$ характеризует выраженность аутоиммунного процесса на этапе появления клинических и лабораторных признаков заболевания у всех пациентов с АИТ.

Ключевые слова: аутоиммунный тиреоидит, сахарный диабет 1 типа, антитела, иммуноглобулины, тиреотропный гормон.
\end{abstract}

Вклад авторов: Гирш Я.В. - анализ и интерпретация полученных данных, написание и редактирование текста рукописи, утверждение рукописи для публикации; Болотская Л.А. - разработка концепции и дизайна исследования, обработка материала, анализ и интерпретация полученных данных; Тарлюн А.А. — сбор клинического материала, обработка, анализ материала.

Конфликт интересов: авторы заявляют об отсутствии возможных конфликтов интересов.

Для цитирования: Гирш Я.В., Болотская Л.А., Тарлюн А.А. Сравнительная характеристика клинико-иммунологического статуса пациентов с аутоиммунным тиреоидитом и его сочетанием с сахарным диабетом 1 типа. Доктор.Ру. 2020; 19(2): 45-50. D0I: 10.31550/1727-23782020-19-2-45-50

\section{Comparison of the Clinical Status and Immunological Parameters of Patients with Autoimmune Thyroiditis with and without Type 1 Diabetes Mellitus}

Ya.V. Girsh, L.A. Bolotskaya, A.A. Tarlyun

Surgut State University (a Khanty-Mansiysk Autonomous District-Yugra Government-funded Institution of Higher Education); 22 Energetikov St., Surgut, Russian Federation 628408

\section{ABSTRACT}

Study Objective: To assess and compare the condition of patients who have autoimmune thyroiditis (AIT) with those who have AIT plus type 1 diabetes mellitus (T1DM), based on a combination of clinical, hormonal, and immunological markers.

Study Design: This was an open-label, comparative, parallel-group study.

Materials and Methods: One hundred and seventy-five patients with a verified diagnosis of AIT were enrolled in the study. Group 1 consisted of patients with AIT only, and Group 2 was made up of patients with both AIT and T1DM. Comprehensive analysis of medical-history data

Гирш Яна Владимировна (автор для переписки) - профессор кафедры детских болезней БУ ВО ХМАО-Югры СурГУ, д. м. н., профессор. 628408, Россия, 2. Сургут, ул. Энергетиков, д. 22. eLIBRARY.RU SPIN: 6683-8810. E-mail: yanaef@yandex.ru

Болотская Лариса Алексеевна - д. м. н., профессор кафедры патофизиологии и общей патологии БУ ВО ХМАО-Югры СурГУ. 628408, Россия, 2. Сургут, ул. Энергетиков, д. 22. eLIBRARY.RU SPIN: 5106-7357. E-mail: labolotskaya@mail.ru

Тарлюн Александра Александровна - аспирант кафедры патофизиологии и общей патологии БУ ВО ХмАО-Югры СурГУ. 628408, Россия, 2. Сургут, ул. Энергетиков, д. 22. E-mail: tarlyun@mail.ru 
and clinical signs was done, as well as measurement of the following parameters: autoantibodies (autoAb) to thyroglobulin (Tg), thyroid peroxidase (TP0), thyroid-stimulating hormone (TSH) receptor, thyroid hormones, and insulin; and blood serum immunoglobulins.

Study Results: Comprehensive examination of patients with AIT and manifest hypothyroidism revealed direct correlations between anti-TPO, anti-insulin, and anti-Tg autoAb and TSH levels, which were stronger in the group of patients with both AIT and T1DM. A correlation was found between an increase in TSH levels and a rise in serum immunoglobulin (IgG, IgM, and IgA) concentrations, which lawfully was stronger in the group of patients with both AIT and T1DM. An assessment of cellular immunity in the study participants showed an inverse correlation between TSH levels and levels of T-helpers (CD3+/CD4+) and T-cytotoxic cells (CD3+/CD8+), which was stronger in patients with both AIT and T1DM. In this group, a significant increase in CD3+/CD16/56+ levels also directly correlated with TSH levels.

Conclusion: Hypothyroidism inevitably stimulates the production of autoAb to a number of protein substances. Reduced levels of CD3+/CD4+ and $C D 3+/ C D 8+$ are explained by their shift to areas of autoimmune inflammation, while significantly higher absolute and relative numbers of $\mathrm{CD} 3+/ \mathrm{CD} 16 / 56$ + reflect the intensity of the autoimmune process at the time of clinical and laboratory manifestation in all AIT patients. Keywords: autoimmune thyroiditis, type 1 diabetes mellitus, antibodies, immunoglobulins, thyroid-stimulating hormone.

Contribution: Girsh, Ya.V. - data processing analysis and interpretation and assistance in writing and editing the final manuscript, approval of the manuscript for publication; Bolotskaya, L.A. - the concept and design of the study, data processing analysis and interpretation; Tarlyun, A.A. clinical data collection, assistance in information processing and analysis.

Conflict of interest: The authors declare that they do not have any conflict of interests.

For citation: Girsh Ya.V., Bolotskaya L.A., Tarlyun A.A. Comparison of the Clinical Status and Immunological Parameters of Patients with Autoimmune Thyroiditis with and without Type 1 Diabetes Mellitus. Doctor.Ru. 2020; 19(2): 45-50. (in Russian) DOI: 10.31550/1727-2378-2020-19-2-45-50

\section{ВВЕДЕНИЕ}

Аутоиммунные заболевания щитовидной железы возникают в результате нарушения в организме регуляции иммунной системы, что приводит к иммунной атаке на саму щитовидную железу. Это заболевания, связанные с развитием опосредованных Т-клетками органоспецифических аутоиммунных нарушений. Наиболее распространен в настоящее время аутоиммунный тиреоидит (АИТ), который характеризуется наличием циркулирующих антител и инфильтрацией аутореактивными лимфоцитами щитовидной железы, а в ряде случаев и орбиты [1-4].

Частота АИТ составляет около 5\%, однако распространенность носительства антитиреоидных аутоантител (АутоАТ) в популяции значительно выше. Вопрос о роли АутоАТ к компонентам собственных тканей или внутриклеточным ферментам в развитии АИТ достаточно сложен, т. к. динамика их уровней не имеет четкого диагностического и прогностического значения для оценки развития и прогрессирования АИТ. Неизвестно, когда именно носительство АутоАТ переходит в заболевание, всегда это происходит или носительство остается на протяжении всей жизни без возникновения болезни [5].

Нередко обнаруживается иммунологическое совпадение с другими аутоиммунными болезнями и семейным аутоиммунным анамнезом. Существует генетическая предрасположенность к нескольким аутоиммунным патологиям [6]. Из всех вариантов сочетаний аутоиммунных заболеваний наиболее часто дифференцируются СД 1 типа (СД1) и аутоиммунные заболевания щитовидной железы $[7,8]$. Существуют ассоциации между АИТ и другими болезнями, системными аутоиммунными нарушениями (СД, ревматоидным артритом, системной красной волчанкой, саркоидозом, псориатическим артритом) [9].

При АИТ и других аутоиммунных расстройствах гуморальные и клеточные иммунные механизмы тесно связаны между собой и имеют перекрестную реакцию. В связи с этим гуморальные и клеточные иммунные механизмы взаимно усиливают ответные реакции, определяя сложные механизмы патогенеза АИТ [10, 11]. Вследствие взаимоотягощающего влияния аутоиммунных заболеваний друг на друга требуется более тщательное наблюдение за пациентами с АИТ и другой аутоиммунной патологией с целью выявления прогрессирования заболевания и ранней диагностики других аутоиммунных нарушений.
Цель исследования: на основании комплексного определения клинических, гормональных и иммунологических маркеров дать сравнительную оценку статуса пациентов с АИТ и АИТ в сочетании с СД1.

\section{МАТЕРИАЛЫ И МЕТОДЫ}

Проведено открытое сравнительное исследование с параллельными группами на базе БУ ВО ХМАО-Югры «Сургутский государственный университет», БУ ХМАО-Югры «Нижневартовская городская поликлиника» в период 2013-2018 гг.

В исследование включены 175 пациентов с верифицированным диагнозом АИТ, согласно Клиническим рекомендациям по диагностике и лечению АИТ [12]. Возраст пациентов - 18-55 лет.

Участники были разделены на две группы в зависимости от наличия или отсутствия СД1: группа 1 - пациенты только с АИТ $(n=150)$, группа $2-$ с АИТ и СД1 $(n=25)$. Больные АИТ и СД1 были моложе, чем лица только с АИТ (табл. 1).

Группу контроля (n = 30) составили практически здоровые лица (доноры крови) без аутоиммунной патологии в анамнезе и на момент включения в исследование, с уровнями ТТГ, антител к ТП0, к ТГ в пределах референсных значений, без острых, хронических заболеваний в фазе обострения.

Для решения поставленных задач проведены клинические, лабораторные исследования. Всем пациентам проводился забор крови натощак из периферической вены для биохимического и гормонального исследований.

Гормональное исследование сыворотки крови осуществляли на иммунохимическом анализаторе Access 2 Immunoassay System (Beckman Coulter, США). АутоАТ к ТГ, ТП0, инсулину, к рецептору ТТГ (рТТГ) определяли с помощью ИФА на стандартных плашках по методике «БиоХимМак» (Россия). Оптическую плотность оценивали на спектрофотометре Reader Pasteur LP 400 (Франция) с длиной волны 450 нм, по результатам которой строили калибровочную кривую.

Определение Ig классов A, M, G в сыворотке крови производили на стандартных плашках нефелометром NEPHELOstar Plus (BMG LABTECH, Германия) нефелометрическим методом с помощью стандартных антииммуноглобулиновых сывороток («МедБиоСпектр», Россия). Популяционный состав лимфоцитов, субпопуляционный состав Т-клеток исследован по стандартной методике на проточном цитометре Beckman 
Coulter Epics XL Flow Cytometer (Beckman Coulter, США) с помощью моноклональных антител.

УзИ щитовидной железы делали на ультразвуковом сканеpe ACUSON X300 (Siemens, Германия).

Статистический анализ полученных данных проводили при помощи пакетов статистических программ (Microsoft Access, Excel for Windows, SPSS Statistics 2.0). Количественные показатели представлены в виде Me (L-H), где Me - медиана, L и H - нижний и верхний квартиль соответственно. Статистическую значимость различий между двумя независимыми количественными переменными оценивали с использованием непараметрических критериев Манна - Уитни.

Для исследования наличия и силы связей между изучаемыми параметрами (гормонального профиля щитовидной железы, клеточного и гуморального звеньев иммунитета) применяли коэффициент ранговой корреляции Спирмена. Статистически значимыми считали различия при $\mathrm{p}<0,05$.

Все пациенты в рамках исследования добровольно подписали информированное согласие. Этическая экспертиза протокола исследования проведена в локальном этическом комитете БУ В0 «Сургутский государственный университет».

\section{РЕЗУЛЬТАТЫ И ОБСУЖДЕНИЕ}

Большинство пациентов с АИТ, вошедших в исследование, вне зависимости от наличия/отсутствия СД1 составили лица, проживавшие в условиях северных территорий от 10 до 20 лет (см. табл. 1). В исследуемых группах превалировали женщины в возрасте 26-35 лет. у 8,1\% участников групп 1 и 2 были родственники 1-й, 2-й линии с различными аутоиммунными заболеваниями.

В семьях больных с АИТ (у лиц первой степени родства: родитель - потомок) регистрировали случаи других аутоиммунных заболеваний, частота которых не различалась в группах.

Все 175 пациентов, включенных в исследование, получали заместительную терапию левотироксином натрия (50-75100 мкг/сут). Критерием адекватности назначаемой заместительной терапии в группах 1 и 2 считали поддержание нормальной концентрации ТТГ в сыворотке крови, отсутствие жалоб и клинических проявлений. В группе пациентов с АИТ и СД1 в 100\% случаев проводилась базис-болюсная инсулинотерапия: инсулин гларгин + инсулин аспарт в среднесуточной дозе $25 \pm 7,8$ ЕД/сут и $20 \pm 5,6$ ЕД/сут соответственно. Длительность течения СД1 на момент включения в исследование в группе 2 превышала 1 год и составила до 5 лет у $52 \%$ пациентов и более 5 лет - у $48 \%$.

Пациенты с манифестным гипотиреозом предъявляли разнообразные жалобы, наиболее частые: общая утомляемость и слабость ( $\mathrm{n}=67,38,3 \%)$, перепады настроения ( $n=67,38,3 \%)$, чувство комка в горле $(n=42,24 \%)$. Частота жалоб различалась в исследуемых группах, они значимо чаще имели место у участников группы 1 , чем у больных группы 2: общая утомляемость - у 31\% и 7\% соответственно, снижение работоспособности - у $26 \%$ и 4\%, перепады настроения - у $32 \%$ и 6,3\%, чувство комка

Таблица 1 / Table 1

Общая характеристика пациентов исследуемых групп, n (\%)

Summary of characteristics of patients in the study groups, $\mathrm{n}(\%)$

\begin{tabular}{|c|c|c|c|}
\hline Показатели / Parameters & Bcero / Total & Группа 1 / Group 1 & Группа 2 / Group 2 \\
\hline Общее число пациентов / Total number of patients & $175(100,0)$ & $150(85,7)$ & $25(14,3)$ \\
\hline \multicolumn{4}{|c|}{ Возрастные группы / Age groups } \\
\hline 18-25 лет / 18-25 years old & $25(14,3)$ & $15(10,0)$ & $10(40,0)$ \\
\hline 26-35 лет / $26-35$ years old & $77(44,0)$ & $65(43,3)$ & $12(48,0)$ \\
\hline $36-45$ лет / $36-45$ years old & $49(28,0)$ & $46(30,7)$ & $3(12,0)$ \\
\hline 46-55 лет / 46-55 years old & $24(13,7)$ & $24(16,0)$ & 0 \\
\hline $\begin{array}{l}\text { Пол / Gender: } \\
\text { • мужчины / Male } \\
\text { • женщины / Female }\end{array}$ & $\begin{array}{l}37(21,1) \\
138(78,9)\end{array}$ & $\begin{array}{l}27(18,0) \\
123(82,0)\end{array}$ & $\begin{array}{l}10(40,0) \\
15(60,0)\end{array}$ \\
\hline \multicolumn{4}{|c|}{ Продолжительность жизни на Севере / Duration of living in the North } \\
\hline$<10$ лет / $<10$ years & $30(17,1)$ & $25(16,7)$ & $5(20,0)$ \\
\hline 10-20 лет / 10-20 years & $93(53,2)$ & $76(50,7)$ & $17(68,0)$ \\
\hline$>20$ лет / > 20 years & $52(29,7)$ & $49(32,6)$ & $3(12,0)$ \\
\hline \multicolumn{4}{|c|}{$\begin{array}{c}\text { Наличие в семье родственников 1-й, 2-й линии родства с аутоиммунным заболеванием / } \\
\text { First- and second-degree relatives withan autoimmune disease }\end{array}$} \\
\hline $\begin{array}{l}\text { Аутоиммунная тромбоцитопения / Autoimmune } \\
\text { thrombocytopenia }\end{array}$ & $1(0,6)$ & $1(0,7)$ & 0 \\
\hline Рассеянный склероз / Multiple sclerosis & $1(0,6)$ & 0 & $1(4,0)$ \\
\hline Ревматоидный артрит / Rheumatoid arthritis & $4(2,3)$ & $1(0,7)$ & $3(12,0)$ \\
\hline Болезнь Аддисона / Addison's disease & $8(4,6)$ & $8(5,3)$ & 0 \\
\hline \multicolumn{4}{|c|}{ Соnутствующие заболевания / Concomitant diseases } \\
\hline Гипертоническая болезнь / Hypertensive disease & $37(21,1)$ & $32(21,3)$ & $5(20,0)$ \\
\hline Ожирение / Obesity & $48(27,4)$ & $48(32,0)$ & 0 \\
\hline Хронический холецистит / Chronic cholecystitis & $68(38,9)$ & $52(24,7)$ & $9(36,0)$ \\
\hline
\end{tabular}


в горле - у 17\% и 7\%, ухудшение памяти - у 16\% и 2\%, бессонница - у $25,7 \%$ и $3 \%$; боли в области сердца $(8,5 \%)$ и повышение веса (24\%) были только в группе 1 . В этой группе чаще встречались и другие клинические проявления и жалобы: отеки диагностированы у 9,7\% пациентов, боли в суставах - у 8,5\%, выпадение волос - у $17 \%$. У 4,6\% больных АИТ и СД1 регистрировали жалобы на гипогликемические состояния.

Таким образом, неспецифические жалобы и клинические проявления, характеризующие манифестный АИТ, были менее выражены в группе с сочетанной аутоиммунной патологией (АИТ + СД1).
Оценка функции щитовидной железы в исследуемых группах выявила ожидаемое повышение содержания ТТГ в сравнении с показателями в группе контроля. Уровни свободных фракций Т4 и ТГ соответствовали референсным значениям с тенденцией к показателям у нижней границы нормативного интервала (табл. 2).

Проведена оценка адаптивного иммунитета у пациентов. Изучены концентрации АутоАТ к ТГ, ТПО, к инсулину, собственным компонентам поджелудочной железы. АутоАТ указанных групп зафиксированы у пациентов обеих групп, однако лица с сочетанием АИТ и СД1 имели более высокие показатели (табл. 3). При этом АутоАТ к рТТГ в обеих

Таблица 2 / Table 2 б

Показатели гормонального профиля щитовидной железы у пациентов исследуемых групп, $\mathrm{Me}(\mathrm{L}-\mathrm{H})$

Thyroid hormone profile in patients in the study groups, $\mathrm{Me}(\mathrm{L}-\mathrm{H})$

\begin{tabular}{|l|l|l|l|}
\hline \multicolumn{1}{|c|}{ Показатели / Parameters } & $\begin{array}{c}\text { Группа контроля }(\mathbf{n}=30) / \\
\text { Control group }(\mathbf{n}=30)\end{array}$ & $\begin{array}{c}\text { Группа 1 }(\mathbf{n}=150) / \\
\text { Group 1 }(\mathbf{n}=150)\end{array}$ & $\begin{array}{c}\text { Группа 2 }(\mathbf{n}=25) / \\
\text { Group 2 }(\mathbf{n}=25)\end{array}$ \\
\hline $\begin{array}{l}\text { Свободные фракции трийодтиронина, } \\
\text { пмоль/л / Free triiodothyronine, pmol/L }\end{array}$ & $2,10(2,04-2,33)$ & $2,27(2,10-2,40)^{*}$ & $2,38(2,33-2,46)^{*, \wedge}$ \\
\hline $\begin{array}{l}\text { Свободные фракции тироксина, } \\
\text { пмоль/л / Free thyroxine, pmol/L }\end{array}$ & $1,10(0,85-1,27)$ & $1,15(1,00-1,30)^{*}$ & $0,65(0,61-0,74)^{*, \wedge}$ \\
\hline $\begin{array}{l}\text { Tиреотропный гормон, ME/мл / Thyroid- } \\
\text { stimulating hormone, IU/mL }\end{array}$ & $3,20(3,16-3,26)$ & $11,60(11,50-11,72)^{* *}$ & $10,27(10,45-10,81)^{* *}$ \\
\hline $\begin{array}{l}\text { Tиреоглобулин, ммоль/л / Thyroglobulin, } \\
\text { mmol/L }\end{array}$ & $1,40(1,24-1,46)$ & $1,21(1,14-1,30)^{*}$ & $1,15(1,14-1,23)^{*}$ \\
\hline
\end{tabular}

Примечания.

1. Отличия от группы контроля статистически значимы: $(*)-\mathrm{p}<0,05,(* *)-\mathrm{p}<0,01$.

2. Отличия от группы 1 статистически значимы: $\left(^{\wedge}\right)-\mathrm{p}<0,05$.

Notes:

1. The differences from the control group were statistically significant: $\left(^{*}\right)-\mathrm{p}<0.05,(* *)-\mathrm{p}<0.01$

2. The differences from Group 1 were statistically significant: $\left(^{\wedge}\right)-p<0.05$.

Таблица 3 / Table 3 。

Состояние гуморального звена иммунитета пациентов исследуемых групп, Me (L-H) Humoral immunity parameters of patients in the study groups, $\mathrm{Me}(\mathrm{L}-\mathrm{H})$

\begin{tabular}{|c|c|c|c|}
\hline Показатели / Parameters & $\begin{array}{c}\text { Группа контроля }(n=30) / \\
\text { Control group }(n=30)\end{array}$ & $\begin{array}{c}\text { Группа } 1(n=150) / \\
\text { Group } 1(n=150)\end{array}$ & $\begin{aligned} \text { Группа } 2(n=25) / \\
\text { Group } 2(n=25)\end{aligned}$ \\
\hline $\begin{array}{l}\text { Аутоантитела (АутоАТ) } \\
\text { к тиреоглобулину, ME/мл / } \\
\text { Autoantibodies (autoAb) to thyroglobulin, } \\
\text { IU/mL }\end{array}$ & 0 & $1,5(1,4-1,7)$ & $1,6(1,3-1,8)$ \\
\hline $\begin{array}{l}\text { АутоAТ к тиреопероксидазе, ME/мл / } \\
\text { AutoAb to thyroid peroxidase, IU/mL }\end{array}$ & $6,2(4,1-6,7)$ & $697,5(640,5-791,5)^{*}$ & $790(741-821)^{*}$ \\
\hline $\begin{array}{l}\text { АутоАТ к собственным тканям } \\
\text { поджелудочной железы, ME/мл / } \\
\text { AutoAb to patient's own pancreatic tissue, } \\
\text { IU/mL }\end{array}$ & 0 & $0,19(0,15-0,25)$ & $3,5(3,31-3,62)^{\wedge}$ \\
\hline $\begin{array}{l}\text { АутоAТ к инсулину, ME/мл / AutoAb to } \\
\text { insulin, IU/mL }\end{array}$ & 0 & $3,2(3,0-3,89)^{* *}$ & $7,4(6,19-8,49)^{* * *}$ \\
\hline IgA, г/л / IgA, g/L & $2,1(1,7-2,21)$ & $4,9(4,57-5,21)^{* *}$ & $6,2(5,78-6,44)^{* * *, \wedge}$ \\
\hline IgM, г/л / IgM, g/L & $2,1(1,78-2,21)$ & $1,9(1,4-2,0)$ & $2,8(2,4-3,2)^{\wedge}$ \\
\hline $\mathrm{IgG}$, г/л / IgG, g/L & $11(9-12)$ & $15(13,4-16,4)^{* *}$ & $19(15-22) * *, \wedge \wedge$ \\
\hline
\end{tabular}

Примечания.

1. Отличия от группы контроля статистически значимы: $\left(^{*}\right)-\mathrm{p}<0,001,\left({ }^{* *}\right)-\mathrm{p}<0,05,\left({ }^{* * *}\right)-\mathrm{p}<0,01$.

2. Отличия от группы 1 статистически значимы: $\left(^{\wedge}\right)-\mathrm{p}<0,01,\left({ }^{\wedge}\right)-\mathrm{p}<0,001$.

Notes:

1. The differences from the control group were statistically significant: $\left(^{*}\right)-\mathrm{p}<0.001,\left({ }^{*}\right)-\mathrm{p}<0.05,\left({ }^{* *}\right)-\mathrm{p}<0.01$.

2. The differences from Group 1 were statistically significant: $(\wedge)-p<0.01,(\wedge \wedge)-p<0.001$. 
группах отсутствовали. У больных группы 2 титр АутоАТ к собственным компонентам поджелудочной железы был значимо выше, чем у пациентов группы $1(p<0,01)$.

В обеих группах содержание IgA и IgG оказалось значимо выше в сравнении с показателями группы контроля (см. табл. 3), значимое отличие уровня IgM у больных АИТ от контрольных значений не найдено. Концентрации IgA, IgM и IgG были значимо выше в группе пациентов с АИТ и СД1, чем в группе только с АИТ.

В группах 1 и 2 определены взаимосвязи между концентрациями ТTГ и $\operatorname{IgG}(r=0,47, p<0,05$ и $r=0,65, p<0,001$ соответственно), ТТГ и IgA $(r=0,49, p<0,002$ и $r=0,64, p<0,001)$, АутоАТ к ТП0 и IgA $(r=0,61, p<0,001$ и r = 0,81, $p<0,001)$.

Полученные данные свидетельствуют о том, что нарушение регуляции гормонов щитовидной железы с формированием гипотиреоидного состояния неизбежно приводит к активации аутоиммунитета с увеличением продукции АутоАТ к ряду белковых структур клеток и тканей организма. Однако данное увеличение является уже результатом активации процессов клеточного иммунитета, которые стартовали задолго до появления клинико-лабораторных изменений.

Значительные изменения наблюдались в иммунном статусе пациентов обеих групп. Абсолютное и относительное количество В-лимфоцитов (CD19/20+) было увеличено В обеих группах (табл. 4). Выявлено значимое снижение относительной и абсолютной концентрации CD3+/CD4+ и $\mathrm{CD} 3+/ \mathrm{CD} 8+$ лимфоцитов на фоне повышения относительного и абсолютного количества CD3+/CD16/56+. Более выраженные отклонения характерны для больных АИТ и СД1 (см. табл. 4).

Полученные результаты показывают вовлечение Т-клеток популяции лимфоцитов в процессы аутодеструкции щито- видной и поджелудочной желез. При протекающих аутоиммунных процессах в обеих железах данные нарушения более выражены у больных с СД1. Снижение содержания $\mathrm{CD} 3+/ \mathrm{CD} 4+$ (Т-хелперов) и $\mathrm{CD} 3+/ \mathrm{CD} 8+$ лимфоцитов (Т-цитотоксических) в периферической крови связано с перераспределением этих клеток в очаги аутоиммунного воспаления (щитовидную железу, поджелудочную железу).

Многократное увеличение относительного и абсолютного количества Т-клеточной субпопуляции - Т лимфоцитов-киллеров (CD3+/CD16/56+) - демонстрирует выраженность аутоиммунного процесса на этапе появления начальных клинических и лабораторных признаков, более сильную у пациентов с сочетанием аутоиммунной патологии. При наличии хронического аутоиммунного воспаления субпопуляция этих клеток будет инициировать дальнейшие аутоиммунные реакции не только по гуморальному, но и по клеточному типу, что согласуется с рядом литературных данных $[4,5]$.

Оценка взаимосвязей показателей гормонального профиля щитовидной железы и клеточного звена иммунитета в группе пациентов только с АИТ выявила отрицательные корреляционные связи показателей ТTГ и Т-хелперов и Т-цитотоксических компонентов: ТТГ и CD3+/CD4+ $(r=-0,51, p<0,001)$ и ТТГ и CD3+/CD8+ $(r=-0,55$, $\mathrm{p}<0,001)$. В группе пациентов с АИТ и СД1 определены аналогичные связи: ТТГ и CD3+/CD4+ $(r=-0,50, p<0,02)$, TTГ и CD3+/CD8+ $(r=-0,48, p<0,02)$. Так как у больных с АИТ и СД1 наблюдалось значительное повышение количества лимфоцитов CD3+/CD16/56+, в этой группе определена его сильная положительная корреляционная связь с уровнем ТТГ $(r=0,62, p<0,001)$. Полученные данные могут свидетельствовать о вовлечении Т-клеток популяции

Таблица 4 / Table 4 ?

Состояние клеточного звена иммунитета пациентов исследуемых групп, Me (L-H) Cellular immunity parameters of patients in the study groups, $\mathrm{Me}(\mathrm{L}-\mathrm{H})$

\begin{tabular}{|c|c|c|c|}
\hline Показатели & $\begin{array}{c}\text { Группа контроля }(\mathrm{n}=30) / \\
\text { Control group }(\mathrm{n}=30)\end{array}$ & $\begin{array}{c}\text { Группа } 1(n=150) / \\
\text { Group } 1(n=150)\end{array}$ & $\begin{array}{c}\text { Группа } 2(n=25) \\
\text { Group } 2(n=25)\end{array}$ \\
\hline $\begin{array}{l}\text { Лейкоциты, абс. / White blood } \\
\text { cells, absolute count }\end{array}$ & $6000(5500-6400)$ & $5500(5450-5870)$ & $5524(5315-5985)$ \\
\hline $\begin{array}{l}\text { Лимфоциты, \% / Lymphocytes, \% } \\
\text { кл/мкл / cells//L }\end{array}$ & $\begin{array}{l}32,5(25-39) \\
2000(1500-2450)\end{array}$ & $\begin{array}{l}38(35-41,5) \\
2160(1941-2276)\end{array}$ & $\begin{array}{l}36,9(34-40) \\
2031(1806-2390)\end{array}$ \\
\hline 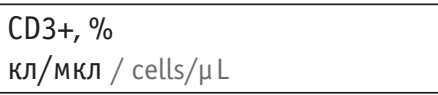 & $\begin{array}{l}71,5(68-75) \\
1400(1100-1600) \\
\end{array}$ & $\begin{array}{l}68(66-69) \\
1469(1296-1571) \\
\end{array}$ & \begin{tabular}{|l|}
$69(67-71)$ \\
$1402(1211-1697)$ \\
\end{tabular} \\
\hline $\begin{array}{l}\text { CD3+CD4+, \% } \\
\text { кл/мкл / cells/ } \mu \mathrm{L}\end{array}$ & $\begin{array}{l}42(39-45) \\
900(800-1000) \\
\end{array}$ & $\begin{array}{l}36(36-37)^{*} \\
515(472-581)^{*}\end{array}$ & $\begin{array}{l}30,5(27,5-32)^{* *, \wedge} \\
428(332-543)^{*}\end{array}$ \\
\hline 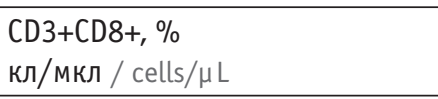 & $\begin{array}{l}35,5(32-38) \\
700(600-800) \\
\end{array}$ & $\begin{array}{l}27(25-29) * * \\
383(355-431)^{*}\end{array}$ & $\begin{array}{l}25(17,5-33) *, \wedge \wedge \\
350(328-400) *, \wedge \\
\end{array}$ \\
\hline 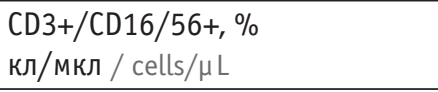 & $\begin{array}{l}3,5(2,2-4,8) \\
90(85-95) \\
\end{array}$ & $\begin{array}{l}11(10-11,5)^{* *} \\
233(223-250)^{*}\end{array}$ & $\begin{array}{l}39,5(34,5-45,5)^{*}, \wedge \wedge \\
554(419-772) *, \wedge \wedge\end{array}$ \\
\hline 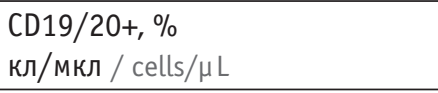 & $\begin{array}{l}13(10-15) \\
300(220-380) \\
\end{array}$ & $\begin{array}{l}19(18-21)^{* *} \\
350(230-410)\end{array}$ & $\begin{array}{l}19,5(18-20)^{* *} \\
396(326-478)\end{array}$ \\
\hline $\begin{array}{l}\text { Иммунорегуляторный индекс / } \\
\text { Immunoregulatory index }\end{array}$ & $2(1,6-2,4)$ & $1,4(1,3-1,5)$ & $1,2(0,8-1,9)^{* *}$ \\
\hline
\end{tabular}

Примечания.

1. Отличия от группы контроля статистически значимы: $\left(^{*}\right)-\mathrm{p}<0,01,(* *)-\mathrm{p}<0,05$.

2. Отличия от группы 1 статистически значимы: $\left(^{\wedge}\right)-\mathrm{p}<0,05,\left({ }^{\wedge \wedge}\right)-<0,01$.

Notes:

1. The differences from the control group were statistically significant: $\left(^{*}\right)-\mathrm{p}<0.01,(* *)-\mathrm{p}<0.05$.

2. The differences from Group 1 were statistically significant: $\left(^{\wedge}\right)-\mathrm{p}<0.05,\left(^{\wedge \wedge}\right)-<0.01$. 
лимфоцитов в процессы аутодеструкции щитовидной и поджелудочной желез.

\section{ЗАКЛЮЧЕНИЕ}

При непрерывно протекающем аутоиммунном процессе нарушения более выражены у пациентов с сочетанной аутоиммунной патологией - аутоиммунным тиреоидитом (АИТ) и СД 1 типа. Значительное возрастание относительного и абсолютного количества Т-клеточной субпопуляции Т-киллеров (CD3+/CD16/56+) - характеризует выраженность аутоиммунного процесса у всех пациентов с АИТ, особенно у больных более молодого возраста с сочетанием аутоиммунных заболеваний, что согласуется с данными других исследований $[13,14]$.

Результаты корреляционного анализа позволяют предположить, что имеющаяся тиреоидная недостаточность определяет негативное действие на состояние клеточных и гумо-

\section{ЛИТЕРATУPA / REFERENCES}

1. Caturegli P., De Remigis A., Chuang K., Dembele M., Iwama A. Iwama S. Hashimoto's thyroiditis: celebrating the centennial through the lens of the Johns Hopkins hospital surgical pathology records. Thyroid. 2013; 23(2): 142-50. DOI: 10.1089/thy. 2012.0554

2. Болезни щитовидной железы: пер. с англ. под ред. Л.И. Бравермана. М.: Медицина, 2000. 432 c. [[Thyroid disorders: translated from English, Braverman L.E., ed. M.: Meditsina; 2000. 432 p. (in Russian)]

3. Kristensen $B$. Regulatory $B$ and $T$ cell responses in patients with autoimmune thyroid disease and healthy controls. Dan. Med. J. 2016; 63(2). PII: B5177.

4. Pyzik A., Grywalska E., Matyjaszek-Matuszek B., Roliński J. Immune disorders in Hashimoto's thyroiditis: what do we know so far? J. Immunol. Res. 2015; 2015: 979167. DOI: 10.1155/2015/979167

5. Здор В.В. Взаимосвязь гормональной и цитокиновой регуляции при аутоиммунном тиреоидите. Клиническая и экспериментальная тиреоидология. 2017; 13(2): 45-56. [Zdor V.V. Correlation of hormonal and cytokines regulation in case of autoimmune thyroiditis. Clinical and Experimental Thyroidology. 2017; 13(2): 45-56. (in Russian)]. D0I: 10.14341/ ket2017245-56

6. Beato-Vibora P.I., Alejo-González S. Avoiding misdiagnosis due to antibody interference with serum free thyroxin. Int. J. Endocrinol. Metab. 2017; 15(1): e37792. DOI: 10.5812/ijem. 37792

7. Гирш Я.В., Кузнецова Е.С., Кияев А.В. Структура тиреиодной патологии в популяции детей и подростков, проживающих в условиях зобной эндемии северных территорий по результатам ультразвуковых исследований. Клиническая и экспериментальная тиреоидология. 2009; 5(4): 47-53. [Girsh Y.V., Kuznetsova E.S., Kiyaev A.V. Structure of a thyroid gland pathologies in a population of children and the teenagers living in conditions endemic goiter of northern territories by results of ultrasonic researches. Clinical and Experimental Thyroidology. 2009; 5(4): 47-53. (in Russian)]

8. Huber A., Menconi F., Corathers S., Jacobson E.M., Tomer Y. Joint genetic susceptibility to type 1 diabetes and autoimmune thyroiditis: ральных факторов аутоиммунного воспаления, формируя многочисленные взаимосвязи, усугубляющие действие факторов воспаления.

В нашем исследовании у пациентов, имеющих родственников с аутоиммунными заболеваниями, были более значимые изменения тиреоидного профиля, а также титров аутоантител к ряду поверхностных и внутренних структур клеток органов эндокринной системы, сывороточных иммуноглобулинов, чем у участников без отягощенного семейного анамнеза [15]. Для хронического АИТ характерно длительное малосимптомное течение, в связи с этим выявление аутоиммунной патологии у члена семьи определяет необходимость повышенной настороженности для ранней диагностики аутоиммунных заболеваний.

По объективным причинам группа 2 исследования представлена ограниченным числом пациентов, что могло привести к некоторому смещению результатов.

from epidemiology to mechanisms. Endocr. Rev. 2018; 29(6): 697725. DOI: $10.1210 /$ er.2008-0015

9. Zhao C., Gao Y., Zhao L., Li Y., Zhang Y., Wang S. et al. The expression and function of the neonatal FC receptor in thyrocytes of Hashimoto's thyroiditis. Int. Immunopharmacol. 2017; 44: 53-60. DOI: 10.1016/j.intimp.2016.12.032

10. Рымар О.Д., Пьянкова А.К., Максимов В.Н., Мустафина С.В. Семейные случаи аутоиммунных заболеваний щитовидной железы. Клиническая и экспериментальная тиреоидология. 2013; 9(4): 39-45. [Rymar O.D., Pyankova A.K., Maksimov V.N., Mustafina S.V. Family history of autoimmune thyroid disease. Clinical and Experimental Thyroidology. 2013; 9(4): 39-45. (in Russian)]. DOI: 10.14341/ket20139439-45

11. Nazarpour S., Tehrani F. R., Simbar M., Tohidi M., AlaviMajd H., Azizi F. Comparison of universal screening with targeted high-risk case finding for diagnosis of thyroid disorders. Eur. J. Endocrinol. 2016; 174(1): 77-83. DOI: 10.1530/EJE-15-0750

12. Дедов И.И., Мельниченко Г.А., Герасимов Г.А., Фадеев В.В. Петунина Н.А, Александрова Г.Ф. и др. Клинические рекомендации Российской ассоциации эндокринологов по диагностике и лечению аутоиммунного тиреоидита у взрослых. М.; 2013. [Dedov I.I., Melnichenko G.A., Gerasimov G.A., Fadeev V.V., Petunina N.A., Alexandrova G.F. et al. Clinical guidelines of the Russian Endocrinology Association for the diagnosis and treatment of autoimmune thyroiditis in adults. M.; 2013. (in Russian)]

13. Fehérvari Z., Sakaguchi S. CD4+ Tregs and immune control. J. Clin. Invest. 2004; 114(9): 1209-17. DOI: 10.1172/JCI23395

14. Hou H.F., Jin X., Sun T., Li C., Jiang B.F., Li Q.W. Cytotoxic $T$ lymphocyte-associated antigen 4 gene polymorphisms and autoimmune thyroid diseases: an updated systematic review and cumulative meta-analysis. Int. J. Endocrinol. 2015; 2015: 747816. DOI: $10.1155 / 2015 / 747816$

15. Schultheiss U.T., Teumer A., Medici M., Li Y., Daya N., Chaker L. et al. A genetic risk score for thyroid peroxidase antibodies associates with clinical thyroid disease in community-based populations. J. Clin. Endocrinol. Metab. 2015; 100(5): E799-807. DOI: 10.1210/ jc.2014-4352 D 